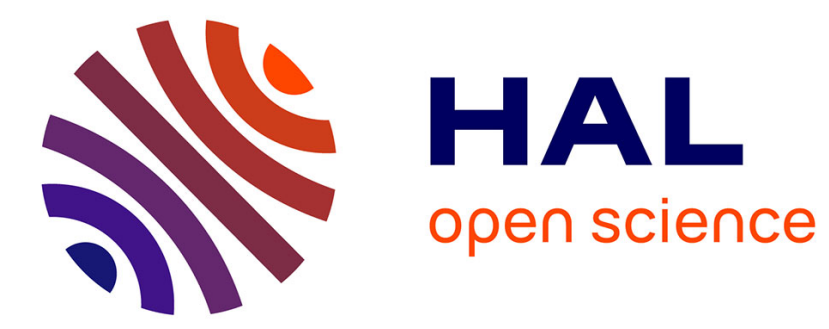

\title{
Les villes dans l'espace social
}

Fabienne Provendier, Denise Pumain, Thérèse Saint-Julien

\section{To cite this version:}

Fabienne Provendier, Denise Pumain, Thérèse Saint-Julien. Les villes dans l'espace social. Travaux de l'Institut de Géographie de Reims, 1982, 51-52, pp.31-44. halshs-01520557

\section{HAL Id: halshs-01520557 https://shs.hal.science/halshs-01520557}

Submitted on 10 May 2017

HAL is a multi-disciplinary open access archive for the deposit and dissemination of scientific research documents, whether they are published or not. The documents may come from teaching and research institutions in France or abroad, or from public or private research centers.
L'archive ouverte pluridisciplinaire HAL, est destinée au dépôt et à la diffusion de documents scientifiques de niveau recherche, publiés ou non, émanant des établissements d'enseignement et de recherche français ou étrangers, des laboratoires publics ou privés. 


\title{
LES VILLES DANS L'ESPACE SOCIAL \\ Fabienne PROVENDIER \\ Denise PUMAIN \\ Thérèse SAINT-JULIEN
}

Université de Paris $I$.

\begin{abstract}
Mots-clés : Aménagement - Géographie sociale - Géographie urbaine - Modèles.
Résumé.- L'étude des profils sociaux des villes révèle des inégalités marquées entre les villes. Ces inégalités sont dues aux spécialisations fonctionnelles héritées de la première révolution industrielle, aux divisions récentes du travail selon la qualification des emplois. Elles dépendent aussi de la place des villes dans la hiérarchie urbaine. L'aménagement doit tenir compte de ces données.

Key words : Models - Planning - Social geography - Urban geography.

Abstract : Cities in social space.- The study of urban social profiles shows pronounced inequalities between cities. The reasons of the inequalities are the functional specializations inherited from the first industrial revolu-

tion, the recent divisions of labour according to the qualification of jobs.

They depend also on the place of towns in urban hierarchy. Planning must ta-

ke these data into account.
\end{abstract}

La connaissance de la structure sociale des villes est liée aux questions d'aménagement géographique, dans la mesure oũ les disparités des niveaux et des modes de vies des populations résidentes s'attachent aux lieux et deviennent des indicateurs d'inégalités territoriales. Les contrastes entre les profils sociaux des agglomérations, depuis longtemps mis en évidence et analysés (Chatelain, 1956), résultent de véritables processus de ségrégation sociale interurbaine. Par quels mécanismes cette ségrégation est-elle engendrée et entretenue à l'échelon géographique d'un ensemble national de villes ? Cet article tente de les repérer à partir de l'observation des profils sociaux d'un très grand nombre de villes (1), diverses par leur taille, leur localisation et leurs fonctions. L'analyse a pour guide les hypothèses suivantes :

- les mécanismes essentiels de la ségrégation sociale interurbaine relèvent des modalités successives de la spécialisation fonctionnelle des villes ainsi que des formes de la division spatiale du travail.

(1) L'échantillon étudié comprend les 409 unités urbaines françaises qui avaient plus de 10.000 habitants en 1975. 
- les ségrégations anciennes résultent davantage des diverses répartitions géographiques des branches d'activité, tandis que les formes plus récentes de ségrégation sociale interurbaine, apparues au cours des trois derniēres décennies, sont l'expression de nouvelles divisions spatiales du travail en termes de qualification des emplois (Lipietz, 1980).

- ce phénomène d'accroissement des inégalités sociales dépendrait, non seulement de 1'appartenance régionale des villes, selon un clivage centre-périphérie, mais aussi du niveau où elles se placent dans la hiérarchie urbaine (Aydalot, 1980).

Outre une évaluation de la validité de ces hypothèses et une précision quant à l'importance relative des mécanismes qu'elles invoquent, nous mettrons en évidence d'autres composantes de l'évolution des ségrégations sociales interurbaines : en premier lieu, une certaine autonomie de réponse des sociētés urbaines, qui leur confère une certaine plasticité dans $1^{\prime}$ adaptation aux conditions nouvelles de l'organisation spatiale de l'économie, en second lieu une interférence non négligeable des représentations collectives du territoire.

\section{1 - LE PHÉNOMÈNE DE SÉGRÉGATION SOCIALE INTERURBAiNe}

Nous ne reviendrons pas ici sur la question de l'adéquation des nomenclatures de catégories socio-professionnelles à la description de la structure sociale des villes. Des articles récents ont contribué à éclairer le débat relatif au fondement et au contenu de ces catégories (par exemple Desrosières et Thevenot, 1979). Malgré 1'hétérogénéité de leurs définitions, les catégories socio-professionnelles demeurent des indicateurs, sensibles bien qu'indirects, des grandes inégalités territoriales, en termes de répartition des patrimoines financier et intellectuel, de partage des revenus, du prestige et des pouvoirs dans les populations résidentes.

Quel que soit le niveau d'agrégation des individus que l'on considère (ménages, quartiers urbains, régions, villes), les catégories socio-professionnelles tendent à se regrouper de la même manière, en fonction de leurs caractéristiques ou de leur localisation. Cette configuration très structurée sert d'ailleurs de base aux nomenclatures. Elle répartit les catégories selon deux axes principaux, le premier pouvant être assimilé à une échelle de statut social, largement corrélée avec celle du prestige, du diplôme et des revenus, le second opposant les travailleurs indépendants aux salariés, les "détenteurs de capital économique aux détenteurs de capital culturel" (Desrosières et Thevenot, 1979).

Cette configuration a pu être observée au niveau des individus ou des ménages, dans 1'analyse des goûts et des pratiques culturelles (Bourdieu, 1976 et 1979), ou de leur position dans le marché matrimonial (Desrosières, 1978). A ce niveau, le maintien des grands axes de la structure sociale au cours du temps, malgré des conditions modifiées, s'explique par le jeu de deux mécanismes contradictoires : 1'un est une tendance à la ségrégation, au renforcement des particularités et de l'isolement de chaque catégorie sociale; 1'autre est une tendance à l'homogénéisation de la société, par la mobilité (latérale, ascendante ou descendante) des individus d'une part, et par la diffusion des particularités d'une catégorie dans les autres, d'autre part.

Une configuration très semblable se retrouve à l'échelon des quartiers urbains de certaines villes moyennes (Debonneuil, Gollac, 1978) ou de Paris et de sa banlieue (Lebart, Tabard, 1971). A ce niveau d'agrégation, l'explication de la configuration réside moins dans les méca- 
nismes inter-individuels de reproduction sociale qu'elle ne passe, très largement, par le biais de l'organisation spatiale du marché foncier urbain.

Qu'en est-il aux niveaux d'agrégation plus larges, régional ou interurbain ? Le terme de "ségrégation sociale" n'a pas, ici, le sens d'un isolement plus ou moins complet de catégories sociales en des lieux différents. Il recouvre l'existence et le maintien, au cours du temps, de profondes disparités dans les poids relatifs des catégories sociales, dans 1a composition de la société urbaine, d'une ville à l'autre. Ces disparités s'ordonnent selon une configuration des catégories socio-professionnelles qui ne ressemble qu'en très grossière approximation à celles observées au niveau des individus ou des ménages (cf. figure 1) : le premier axe de la différenciation sociale des villes, plutôt qu'une échelle de statut social, est une relation d'association-exclusion entre les catégories socio-professionnelles associées aux activités économiques industrielles d'une part, et celles associées aux activités tertiaires d' autre part (Pumain, 1976 ; Saint-Julien, 1982). A 1'intérieur de chacun de ces groupes, 1'ordre des catégories est loin de toujours correspondre à une hiérarchie des fonctions ou des situations sociales (figure 1). Quant au deuxième axe de la configuration, s'il isole assez bien,

$$
\text { Figure } 1 \text { - Les grandes composantes socio-professionnelles }
$$

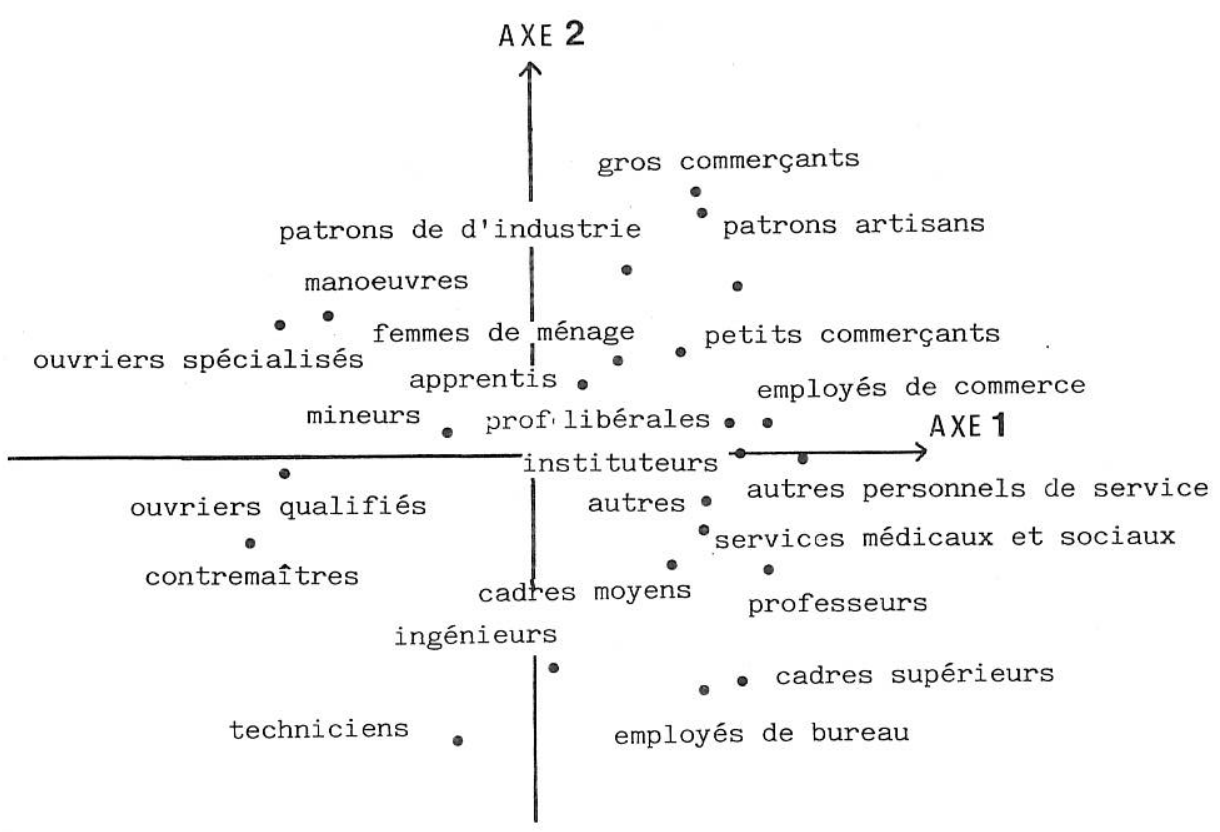

dans l'ensemble, les catégories de travailleurs indépendants de la plupart des catégories salariées, il leur associe aussi les groupes de travailleurs salariés économiquement ou géographiquement dépendants (employés de commerce, personnel de service, manoeuvres, ouvriers spécialisēs) (figure 1).

Géographiquement, la signification du premier axe rejoint celle d'une échelle de statut social dans la mesure où le classement des villes selon cette dimension correspond assez bien à l'ordre des niveaux de revenus dépensés dans les villes (1), ou encore à celui de leur at-

(1) Mesuré par 1'indice de richesse vive de 1'Institut Proscop. 
tractivité relative pour les migrations interurbaines : indicateurs de prestige urbain que nous avons regroupés sous le terme d' "image de marque" des villes (Pumain et Saint-Julien, 1979). Quant au classement selon le deuxième axe de la structure sociale urbaine, il ne recouvre que partiellement un ordre essentiellement défini par le dynamisme du développement économique et que nous avons assimilé à une échelle de "modernitéu des villes.

La logique du regroupement spatial des catégories sociales dans les villes requiert une interprétation spécifique du processus de ségrégation intervenant à cette échelle. Compte tenu des hypothèses formulées ci-dessus, cette analyse doit être effectuée sur un échantillon des villes recouvrant 1 'ensemble du territoire et représentatif des différents niveaux de la hiérarchie urbaine. Nous avons donc choisi de comparer les profils sociaux des 409 villes qui avaient plus de 10.000 habitants en 1975. Jusqu'ici, les tentatives de vérification des hypothèses relatives à l'évolution des modalités de la division spatiale du travail n'ont été effectuées que sur des classes de tailles de villes (Aydalot, 1980), ou seulement pour quelques catégories sociales et sous-ensembles régionaux (Lipietz, 1978).

Comment mesurer le degré de ségrégation des catégories sociales ? La plupart des auteurs utilisent des quotients de localisation, indicateurs des sur ou sous-représentations par rapport à un profil moyen. Cette référence à une situation moyenne ne signifie pas qu'il existe un modèle de société urbaine dont toutes les villes seraient susceptibles de s'approcher. Elle sert tout au plus d'intermédiaire de calcul dans l'identification et l'évaiuation des ségrégations (tableau 1). Nous avons ainsi regroupé les villes en sous-ensembles relativement homogè-

\begin{tabular}{|l|r|r|r|r|}
\multicolumn{8}{|c}{ Tableau 1 - Répartition des catégories sociales dans les villes françaises } \\
\hline
\end{tabular}

Différences significatives au seuil de : ++++ $0,1 \% ;+++0,5 \% ;++1 \% ;+5 \%$

(+ si la valeur augmente avec la taille, - si non). 
nes (3) caractérisés selon le degré de ségrégation des catégories sociales qu'ils concentrent et par la combinaison de ces ségrégations dans les profils sociaux urbains.

\section{2 - LA PERSISTANCE DES SÉGRÉGATIONS HÉRITÉES DE LA PREMIÈrE RÉVOLUTION INDUSTRIELLE}

Aujourd'hui encore, la configuration sociale de l'ensemble des unités urbaines reste marquêe par la grande dichotomie héritée de la première révolution industrielle. Cette forme majeure de ségrégation contribue à isoler de l'ensemble près de 175 unités urbaines, dont l'organisation sociale se caractérise à des degrés divers par une surreprésentation des catégories ouvrières et par une relative faiblesse des catégories sociales les plus favorisēes, salariées surtout.

L'individualité de ces profils sociaux demeure très grande pour toutes les villes qui, à différents moments, ont été le siège du développement d'activités extractives. En dépit du déclin rapide et irréversible de ces activités, les sociētés urbaines qu'elles ont engendrées se perpétuent aujourd'hui sous des formes indissociables de cette genèse très particulière dans 25 agglomérations (figure 2-1). Il est possible qu'une revalorisation de l'exploitation de certains bassins contribue à prolonger ces "vocations" sociales, qui tendent par ailleurs à se maintenir régionalement au-delà des conditions très spécifiques d'expansion dont elles sont l'héritage.

Les modèles sociaux urbains initialement façonnés par la diffusion de la première révolution industrielle $n^{\prime}$ ont pas tous ce caractère d'extrême spécificité. Ils transparaissent pourtant dans les profils d'au moins 154 agglomérations (figures 2-2 et 2-3). Ces profils témoignent de la permanence d'une surreprésentation ouvrière sensible, associées à une sous-représentation marquée des employés, des ingénieurs et des cadres.

Lorsque l'impulsion première et souvent ancienne est imputable à des industries qui avaient de faibles exigences en matière de qualification du travail, on observe que cette faible qualification ouvrière s'est imprimée et s'est pérennisée dans les structures sociales. Dans nombre d'agglomérations, elle a été récemment renforcée par la diffusion, à partir du

(1) Les catégories représentées dans les profils des figures 2 à 4 sont les suivantes :

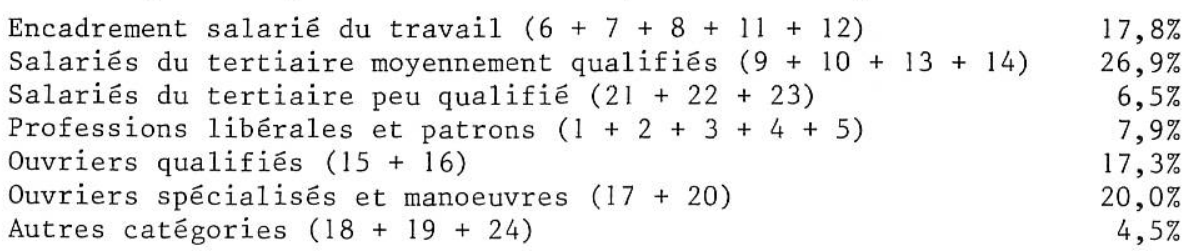

(2) Grandes villes : > 50.000 habitants

(93 dont 47 sont $>100.000$ habitants

Villes moyennes : $20-50.000$ habitants (130)

Petites villes : 10 - 20.000 habitants (186)

(3) Parmi les techniques de regroupement disponibles, nous avons choisi une procédure de classification ascendante hiérarchique utilisant la distance de $\mathrm{X}^{2}$, qui généralise à plusieurs dimensions le calcul des quotients de localisation.

Cette classification a été effectuée sur une nomenclature en 24 catégories socio-professionnelles (source : INSEE), regroupées en sept sous-ensembles pour plus de clarté sur les profils des figures. 
Figure 2 - Tracés des ségrégations de la première révolution industrielle
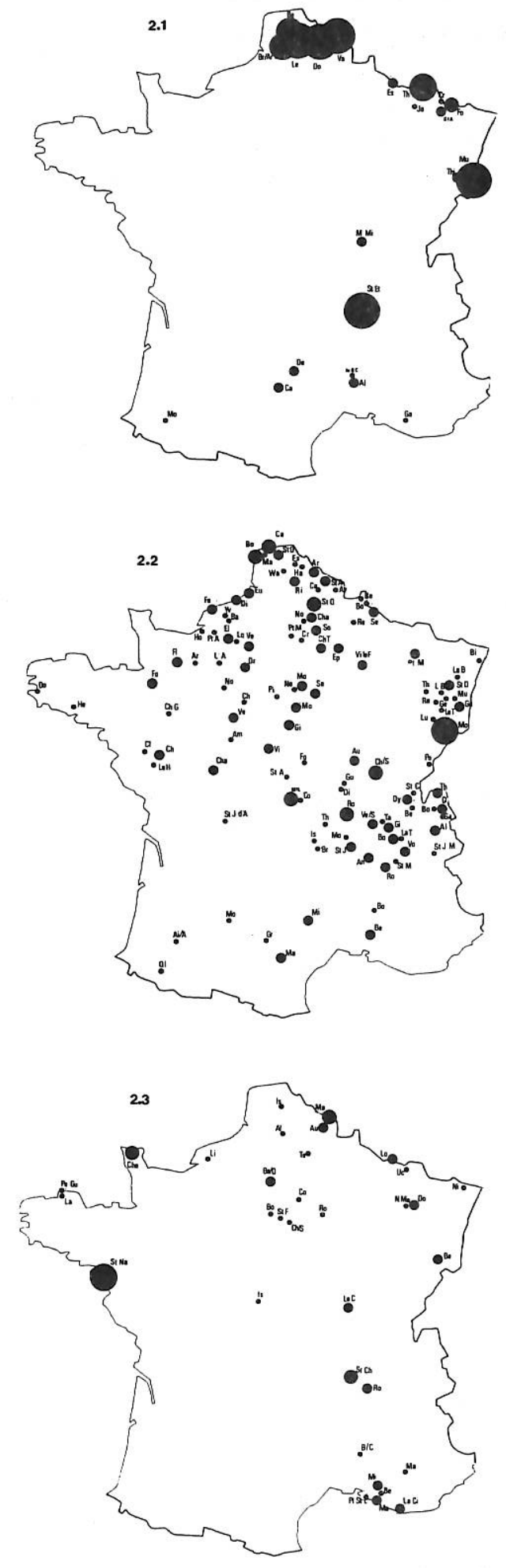

écarts au profil moyen $|x|$

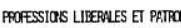

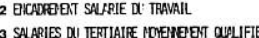

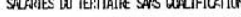

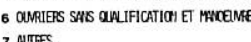
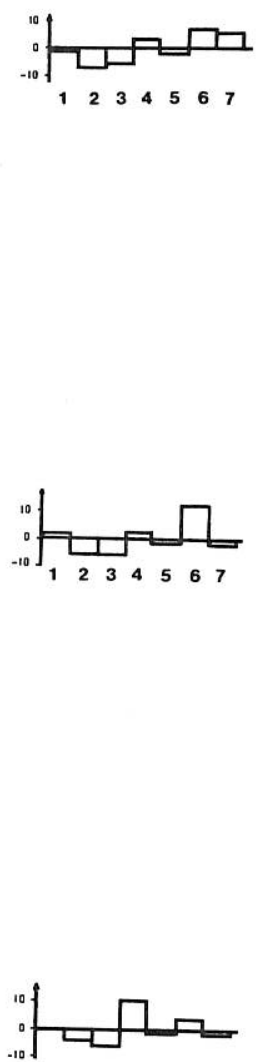

1234567

taille des unités urbaines en milliers d'habitants

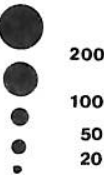


pôle parisien en particulier, d'activités industrielles faisant d'abord appel à une main-d' oeuvre locale banale. On ne s'étonnera pas alors que, sur les 120 agglomérations ainsi caractérisées, 93\% aient moins de 20.000 habitants et $56 \%$ moins de 10.000 habitants, alors que, dans l'ensemble du réseau, ces proportions ne représentent respectivement que 74 et $44 \%$ du nombre total des villes. On ne s'étonnera pas non plus de trouver ici réunies près de la moitié des agglomérations de moins de 20.000 habitants du Bassin Parisien, et près de la moitié de celles de la région du Centre-Est. Ce type de configuration sociale caractérise donc aujourd'hui surtout les petites villes appartenant aux régions qui ont le plus directement bénéficié de la croissance économique récente (figure 2-2).

Quand l'industrialisation initiale a été plutôt soutenue par les industries métallurgiques ou par les industries chimiques, la surreprésentation ouvrière se manifeste, en 1975, au bénéfice des catégories qualifiées (exemples: Maubeuge, Aulnoye, Saint-Nazaire, 34 villes au total). La distribution de ces villes selon la taille est ici beaucoup plus proche de la distribution d'ensemble (figure 2-3).

Cela tendrait à montrer qu'aujourd'hui la concentration relative du travail ouvrier qualifié $n^{\prime}$ est pas de nature à accentuer les distances hiérarchiques entre les villes du réseau. Elle persiste surtout dans les régions touchées par la première révolution industrielle (figure 2-3). En revanche, la pérennisation, voire le renforcement d'une spécificité dans le travail ouvrier peu qualifié, sont plutôt compatibles avec une appartenance aux seuls niveaux inférieurs de la hiérarchie des places centrales, et ce dans les régions qui ont le plus profité de la croissance récente (fig. 2-2).

Les autres agglomérations portent en négatif, mais de manière beaucoup moins marquée, I' empreinte des ségrégations mises en place par la première révolution industrielle : les catégories ouvrières y sont partout plutôt sous-représentées. En dehors de cette caractéristique générale, ces 233 villes témoignent de deux situations nettement distinctes selon le moment de leur intégration : le processus d'intégration à des ensembles géo-économiques de plus en plus vastes s'étant développé depuis un siècle, et progressivement étendu à la totalité du réseau urbain.

\section{3 - VERS UN MODÈLE SOCIAL DES GRANDES VILLES}

Un certain nombre de villes ont été très tôt engagées dans ce processus d'intégration. A des degrés divers, leur histoire récente a renforcé cette participation. Ces agglomérations, qui sont en général d'assez grande taille, possèdent des structures sociales complexes, correspondant à leur position relativement élevée dans la hiérarchie fonctionnelle du réseau. Riches de fonctions diversifiées, elles ont assimilé les effets successifs des processus d'industrialisation, sans que soit brutalement infléchi leur profil social dans le sens d'une surreprésentation extrême des catégories ouvrières. Tout s'est passé comme si différents filtres s'étaient interposés pour, progressivement, individualiser les profils sociaux des plus grandes villes françaises.

En 1975, trois types principaux les caractérisent, qui ne sont que des variations sur un modèle "grandes villes". Le premier concerne, bien entendu, l'agglomération parisienne, qui se démarque toujours très fortement de l'ensemble (figure 3-1) du fait de la surreprésentation 
Figure 3 - Le modèle "Grande Ville"
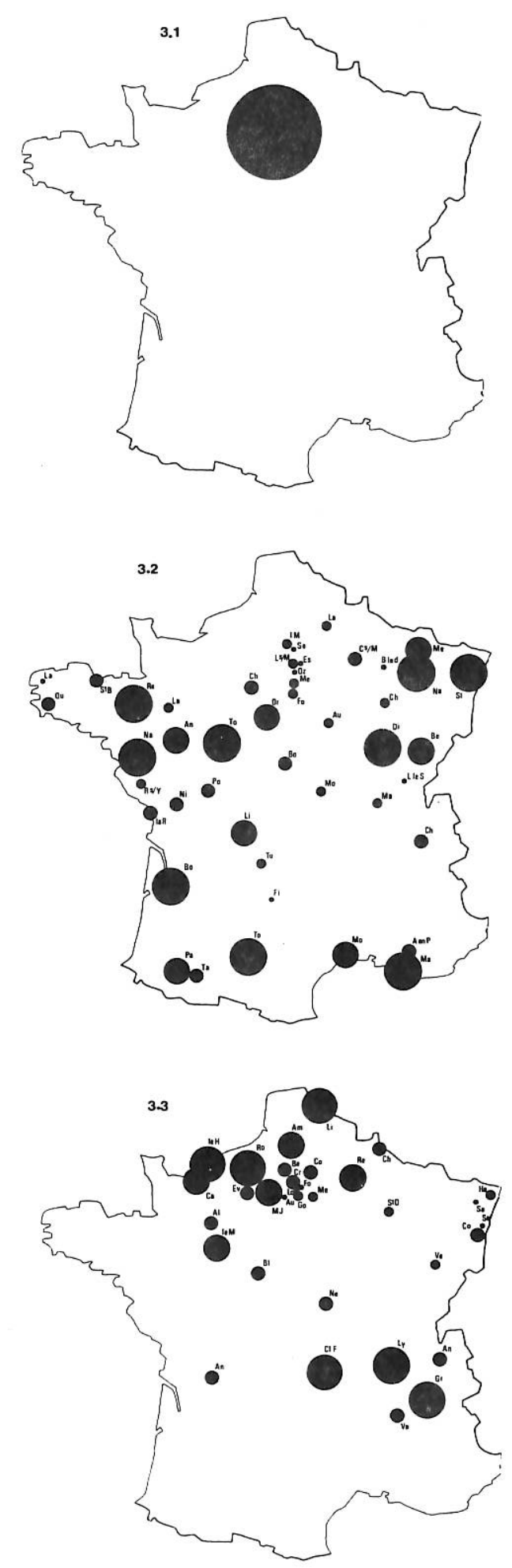

écarts au profil moyenı

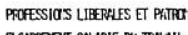

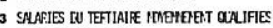

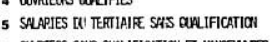

a
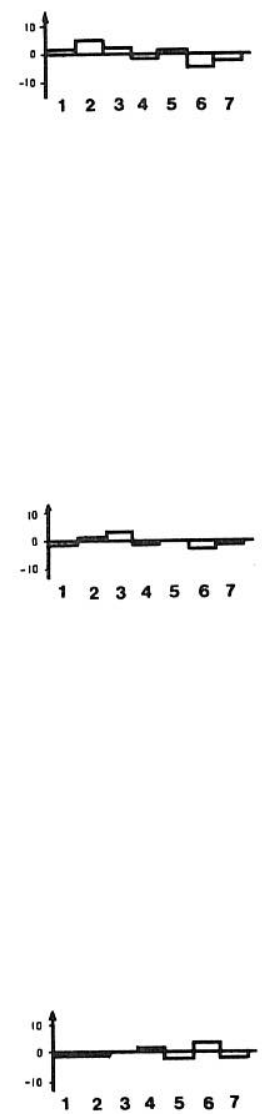

taille des unités urbaines

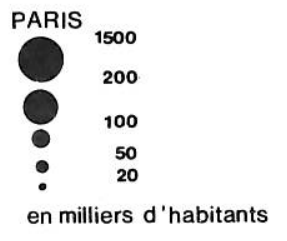


des personnels d'encadrement salariē (ingénieurs, cadres supérieurs et techniciens). L'individualité de la métropole parisienne a été peu altérée par l'évolution récente. Le modèle social des autres grandes villes s'est, sans aucun doute, rapproché du schéma parisien, mais les distances relatives n'ont pas été modifiées de manière sensible.

Le deuxième type du modèle "grandes villes" est très représentatif des profils sociaux des métropoles et capitales régionales. Il est caractérisé (figure 3-2) par une légère surreprésentation des professeurs et des employés sur un profil par ailleurs très proche du profil moyen. Ce type regroupe, certes, un ensemble assez vaste de villes (46 au total). Il est pourtant particulièrement révélateur de la situation des plus grandes unités urbaines. En effet, $35 \%$ des villes concernées ont plus de 100.000 habitants (cette proportion n'est, en 1975, dans l'ensemble du réseau, que de 11\%), 13 sont des capitales de région de programme et 6 des métropoles d'équilibre (Bordeaux, Toulouse, Marseille, Nantes, Strasbourg, Nancy).

La dernière variation du modèle "grandes villes", très proche de la précédente, s'individualise cependant du fait d'une légère surreprésentation des ouvriers spécialisés (figure 3-3). Elle résume bien les profils sociaux d'une quarantaine d'agglomérations, parmi lesquelles on trouve un nombre très significatif de grandes (exemples : Compiègne, Nevers, Blois, Angoulême, etc...) ou très grandes villes (exemples : Li1le, Lyon, Rouen, Grenoble, Caen, Le Mans etc..). Ce type de ville est pratiquement absent des régions de l'Ouest et du Sud-Ouest. Même à ces niveaux supérieurs du réseau urbain, les profils sociaux sont encore infléchis par l'inégal degré d'industrialisation.

Cette disparité apparaît cependant comme atténuée, du fait des rattrapages très sensibles qui se sont opérés depuis trente ans. Les profils sociaux de ces agglomérations se sont rapprochés, contribuant à façonner un modèle français de "grande ville" qui, il y a trente ans, n'était décelable ni dans son contenu ni dans sa configuration géographique actuelle (Pumain et Saint-Julien, 1979). L'émergence de ce modèle social des grandes villes, qui résulte de sédimentations successives, mais qui doit beaucoup à l'évolution récente, tendrait à confirmer 1 'hypothèse d'une ségrégation sociale contemporaine de plus en plus guidée par la hiérarchie du réseau urbain (tableau 1). La trame spatiale des niveaux supérieurs du système actuel des places centrales est, en effet, bien restituée par la distribution géographique des profils sociaux relevant du modèle "grande ville" (figure 3). Certes, l'image obtenue est un peu perturbée par le rattachement de quelques petites villes à ce groupe. Mais, pour l'essentiel, ces agglomérations de moins de 20.000 habitants appartiennent, soit à la région parisienne, soit à sa périphérie immédiate : en région urbaine, le modèle a tendance à se diffuser à des niveaux inférieurs de la hiérarchie, du fait des ségrégations résidentielles permises par les migrations alternantes notamment.

\section{4 - Plusieurs pÉRIPHÉRIES?}

Malgré leurs spécificités tertiaires, les 142 agglomérations regroupées dans un "modèle périphérique" diffèrent très nettement du cas précédent. Elles sont, pour la plupart, de petite ou très petite taille ( $89 \%$ d'entre elles ont moins de 20.000 habitants). Leurs profils sociaux sont, aujourd'hui encore, largement modelés par le maintien des catégories de patrons, de l'artisanat, de l'industrie et du commerce. L'encadrement salarié y est toujours nettement plus réduit qu'ailleurs, et les deux tiers d'entre elles ont également un déficit marqué pour 
les qualifiées des salariés du secteur tertiaire. A l'évidence, ces unités urbaines sont, plus longtemps que d'autres, demeurées à 1'écart des processus d'intégration économique développés depuis un siècle. Cet isolement résulte sans doute à la fois de leur position hiérarchique en retrait et d'une situation régionale "périphérique" : la plupart d'entre elles se trouvent, en effet, concentrées dans 1'Ouest (29 agglomérations sur les 43 de moins de 20.000 habitants de cette région) ; dans le Sud-Ouest, oū la proportion est encore plus forte (28 agglomérations sur 38); et dans les régions méditerranéennes (22 sur 30) (figure 4).

Un premier sous-ensemble de villes, où les catégories ouvrières sont très nettement sousreprésentées, est presque entièrement concentré dans les régions de l'Aquitaine et de la Méditerranée, tout en incluant quelques villes plus septentrionales, pour la plupart en situation littorale (fig. 4-1). Tous les niveaux hiérarchiques sont à peu près bien représentés parmi cette cinquantaine de villes. Leurs profils sociaux sont 1'expression d'une forme régionale d'organisation urbaine où la centralité est plutôt commerciale. Autant que cette centralité d'un type particulier, c'est souvent la fonction touristique qui a engendré la ségrégation sociale la plus manifeste dans ce groupe de villes, et qui se traduit par un surcroît des catégories de personnels de service, associé à la surreprésentation majeure des artisans et petits commerçants. De très grandes agglomérations comme Cannes et Nice, des stations balnéaires comme Dinard ou Arcachon, mais aussi des places centrales aux fonctions peu diversifiées et de tailles diverses (Périgueux, Guéret, Pontivy) sont les principaux types urbains regroupés ici. Le rapprochement de ces villes dans un même type de société urbaine amène à préciser le contenu de l'adjectif "périphérique" qui signifie davantage l'écart par rapport à un certain type d'intégration économique, que l'absence de toute forme d'intégration.

76 villes combinent, dans leur profil social, les traces de situations périphériques anciennes et récentes : une proportion importante d'artisans et de petits commerçants les rapproche du type précédent ; un déficit marqué dans les catégories salariées de l'encadrement et du travail tertiaire qualifié les situe à l'écart des formes de centralité que sont la prestation de services et l'administration ; une légère surreprésentation des ouvriers spécialisés correspond soit à la survivance d'industries anciennes utilisant une main-d'oeuvre peu qualifiée (exemples : Cognac, Castres, Lunel), soit à un saupoudrage récent d'établissements le plus souvent décentralisés (exemples :Guingamp, Lisieux, Romorantin, Mayenne). En majorité (près des deux tiers), il s'agit de très petites villes, de moins de 20.000 habitants (fig. 4-2).

Un troisième groupe, enfin, réunit une quinzaine de villes, dont le profil social se distingue par la présence des personnels militaires. Leur situation géographique les place, au sens propre, à la périphérie du territoire, mais ce n'est pas qu'à ce titre qu'elles se trouvent rapprochées des types précédents : leurs structures sociales portent aussi la marque de l'importance du petit commerce, et l'encadrement comme le travail tertiaire qualifié y sont nettement réduits. Casernes (Joigny), arsenaux (Lorient), bases navales (Toulon, Brest) sont à l'origine de la spécificité de ces sociétés urbaines, qui n'est réductible à aucune autre (figure 4-3). 
Figure 4 - Les villes "périphériques"
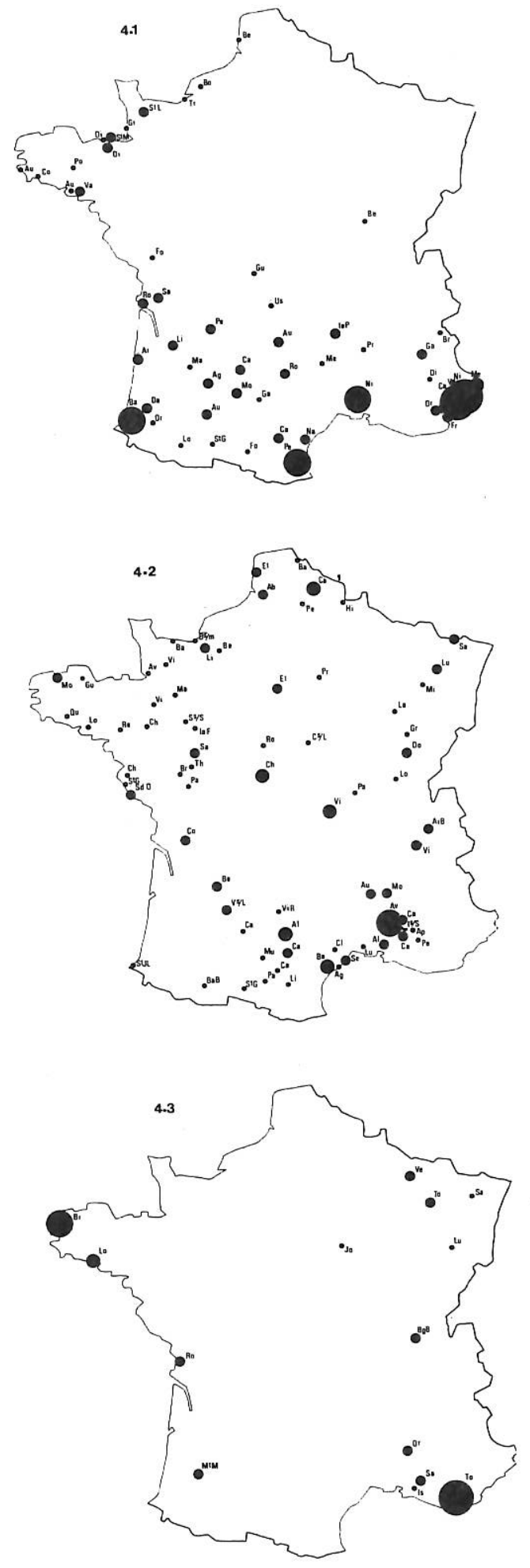

ecarts au profil moyenis:

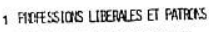

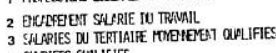

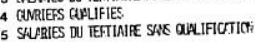

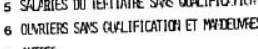

7 ATाE:
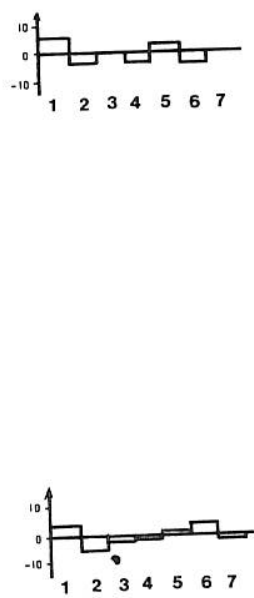

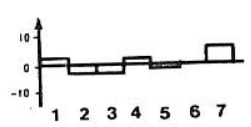

taille des unités urbaines en milliers d'habitants

200
200
0
$\begin{array}{r}100 \\ 20\end{array}$ 


\section{5 - SÉGRÉGATIONS INTERURBAINES ET AMÉNAGEMENT}

Il est probable que, de toutes les caractéristiques urbaines, les sociétés des villes, pourtant en constante évolution, soient les meilleurs témoins des accumulations successives qui ont soutenu le devenir des agglomérations. Le temps long de la succession des grandes innovations qui ont jalonné le développement des pays industrialisés explique, pour une grande part, la diversité des modèles sociaux recensés en 1975, témoins de la nature et de la configuration géographique des ségrégations spatiales qu'ont engendrées ces sédimentations.

On pourrait être tenté d'interpréter les disparités sociales interurbaines, ou plus généralement les décalages interrégionaux qu'elles reflètent, en termes de décalages temporels dans un processus général de développement. Une telle interprétation est acceptable si l'on veut dire que tout le réseau urbain n'a pas bénéficié des mêmes acquis successifs et que certains centres sont restés plus longtemps et plus continuement sur les marges des influences majeures de transformation. On ne doit cependant pas en déduire que, de manière inéluctable, toutes les trajectoires sociales des villes doivent passer par ces étapes successives. Au contraire, on a pu prétendre que la création continue d'inégalités territoriales et leur exploitation étaient une composante nécessaire du développement. Toute la question d'un développement. "équilibré" est de savoir préserver, dans les zones provisoirement moins favorisées par une certaine vague d'innovations, des richesses potentielles susceptibles d'être valorisées par une forme ultérieure de développement (Vining et al. 1982).

Notre présentation des types de sociétés urbaines contemporaines a souligné l'importance des spécialisations économiques dans leur différenciation. Aujourd'hui encore, les plus fortes ségrégations résultent bien des inégales concentrations territoriales des branches d'activité, les ségrégations nouvelles fondées sur la nature et la qualification du travail n'ayant apporté que des variations, certes bien individualisées, sur cette structure fondamentale. Si l'organisation spatiale de l'économie semble déterminante dans la création des ségrégations sociales interurbaines, elle ne suffit pas, toutefois, à expliquer complètement leur maintien, audelà des conditions qui les ont fait naître.

En effet, la diversité des situations urbaines témoigne aussi du degré d'autonomie dont disposent les groupes sociaux dans l'accueil et l'assimilation des forces externes qui tendent à orienter leur transformation. Dans la longue histoire de l'évolution sociale des villes, les spécificités anciennes infléchissent l'assimilation des tendances nouvelles. Cette persistance des sociétés urbaines, dans ce qui fait leur originalité, s'explique en partie par les modalités de leur renouvellement : au moins pour la période contemporaine, on a pu observer que les modifications des profils sociaux des villes étaient sans doute plus dépendantes d'une mobilité sociale interne que des apports externes. La composition sociale des flux migratoires échangés entre les villes et leur environnement tend à reproduire les spécificités de chaque ville, beaucoup plus qu'elle ne rend compte des transformations intervenues dans la société urbaine au cours de la même période (Pumain, 1982). En ce sens, et dans la mesure oũ les courants migratoires dépendent largement de l'information disponible sur les lieux (Courgeau, 1981 ; Claval, 1981), la manière dont les sociétés urbaines sont perçues, leur "image de marque", est un facteur sans doute déterminant de l'inertie du profil social de chaque ville et de la persistance des ségrégations sociales interurbaines au cours du temps. 
Il y a, par ailleurs, tout lieu de penser que le phénomène d'identité collective régionale amplifie ou infléchit la portẻe de ces réactions.

La diversité des profils sociaux urbains doit-elle être déplorée ? Dans une perspective d'aménagement du territoire, la question ne peut être éludée. Au nom de l'équité entre société et espace (Reynaud, 1981), faut-il tendre à homogénéiser les structures sociales urbaines, en essayant de corriger les inégalités dans la répartition territoriale des revenus, de 1'accumulation des richesses, de la culture, de l'initiative et des pouvoirs ? On sait maintenant que l'adaptabilité des groupes sociaux est inversement proportionnelle à leur monolithisme. Le renforcement de spécificités sociales tropétroites diminue donc les chances qu'ont les villes d'accueillir les innovations au moment où, précisément, cet accueil offre l'avantage maximal. Cette observation a été souvent vérifiée quelle que soit la nature de la spécificité sociale en cause. Mais $i l$ y a plus. On sait aussi qu'au-delà de certains seuils bien connus, pour une ville et pour un ensemble régional de villes, la concentration des catégories sociales les plus défavorisées secrète des mécanismes de fermeture accélérant et prolongeant les processus de ségrégation qui ont engendré la concentration. De tels mécanismes rendent, assurément, nécessaires des interventions externes préventives ou, à dēfaut, correctives auprès des villes menacées ou concernées.

Ailleurs, on peut se féliciter du rôle de l'identité régionale. En dépit de son poids mineur au regard des forces externes qui infléchissent les évolutions urbaines, il témoigne que l'accélération de l'intégration économique des régions n'a pas complètement démantelé les réseaux qui maintiennent la cohésion de ce niveau géographique privilégié.

\section{RÉFÉRENCES CITÉES DANS LE TEXTE}

AYDALOT Ph.(1980), Division spatiale du travail et croissance urbaine, Université Paris I, Dossiers du Centre Economie, Espace, Environnement, $\mathrm{n}^{\circ} 12,33 \mathrm{p}$.

BOURDIEU P. (1976), Anatomie du goût, Actes de la Recherche en Sciences Sociales, $n^{\circ} 5$.

BOURDIEU P. (1979), La distinction, Editions de Minuit.

CHATELAIN A. (1956), Géographie sociale des villes françaises en 1946, Revue de Géographie de
Lyon, n², pp. 119-127.

CLAVAL P. (1981), La logique des villes, Paris, L.TEC.

COURGEAU D. (1980), Analyse quantitative des migrations humaines, Paris, Masson.

DEBONNEUIL X., GOLLAC M. (1978), Structure sociale des villes, Economie et Statistiques, $\mathrm{n}^{\circ} 98$,
pp. 51-65. DESROSIERES A. (1978), Marché matrimonial et structure de classes sociales, Actes de la Recherche en Sciences Sociales, n²0-21, pp. 97-107.

DESROSIERES A., THEVENOT L. (1979), Les mots et les chiffres : les nomenclatures socio-professionnelles, Economie et Statistique, $\mathrm{n}^{\circ} 110$, pp. 49-65.

LEBART L., TABARD N. (1971), La morphologie sociale des communes urbaines, Consommation, $\mathrm{n}^{\circ} 14$. LIPIETZ A. (1978), La dimension régionale du développement du tertiaire, Travaux et Recherches
de Prospectives, $\mathrm{n}^{\circ} 75$.

PUMAIN D. (1976), La composition socio-professionnelle des villes françaises, L'Espace Géogra-
phique, $\mathrm{n}^{\circ} 4$, pp. 227-238. PUMAIN D. (1982), Composition des flux migratoires et structure socio-économique des villes,
Communication à la l3ème conférence de I'IARUS, Hongrie, 11-14 mai. PUMAIN D., SAINT-JULIEN T. (1979) Les transformations récentes du système urbain français, $L$,
Espace Géographique, n⿳ 3 , pp. 203-21.1. 
REYNAUD A. (1981), Société, espace et justice, Paris, PUF, 263 p.

SAINT-JULIEN T. (1982), Croissance industrielle et système urbain, Paris, Economica, 280 p. TABARD N., CLAPIER P. (1981), Transformation de la morphologie sociale des communes et variation des consommations, Consommation, $\mathrm{n}^{\circ} 2$, pp. 3-40.

VINING D.R., PALLONNE R. (1982), Migration between peripheral and core regions : a description and tentative explanation of the patterns in 22 countries, Philadelphia, University of Pennsylvanie, Working Papers in Regional Science and Transportation, $80 \mathrm{p}$. 